\title{
Adult congenital heart disease: Cor triatriatum dextrum
}

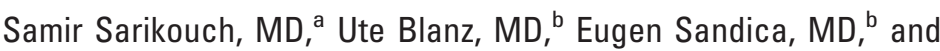 \\ Philipp Beerbaum, MD, ${ }^{a}$ Bad Oeynhausen, Germany
}

\section{Clinical Summary}

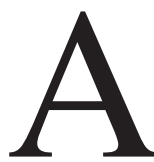

43-year-old man was repeatedly examined at different institutions because of dyspnea on exertion with New York Heart Association class II-III.

On physical examination, there were no signs of chronic heart failure and no heart murmurs $(176 \mathrm{~cm}, 91 \mathrm{~kg}$, and blood pressure of 140/70 mm Hg). Slight central cyanosis was apparent, together with clubbing of the fingers. The hemoglobin level was $18.2 \mathrm{~g} / \mathrm{dL}$, and the transcutaneous oxygen saturation was $92 \%$. Electrocardiography showed no pathology.

Transthoracic echocardiography demonstrated normal-sized ventricles, normal left ventricular ejection, and a mild valvular pulmonary stenosis. Transesophageal echocardiography disclosed an atrial septal defect with bidirectional shunt but no signs of tricuspid regurgitation or stenosis.

Cardiac catheterization ruled out pulmonary hypertension and coronary artery disease. Left-to-right shunt was calculated at a Qp/Qs ratio of 1.27:1. Left atrial oxygen saturation was $93 \%$ on room air.

The patient was referred to our institution for surgical closure of his atrial septal defect. Further diagnostic testing was done because of the thus far unexplained right-to-left shunting. On exercise testing, the capillary $\mathrm{PO}_{2}$ decreased from 64.8 to $48.2 \mathrm{~mm} \mathrm{hg}$, and the maximum oxygen uptake was only $21.5 \mathrm{~mL} \cdot \mathrm{kg}^{-1} \cdot \mathrm{min}^{-1}$, which was about $64 \%$ of the age-related normal value.

Magnetic resonance imaging (MRI) was performed on a $1.5-\mathrm{T}$ Philips scanner using balanced turbo field echo cine sequences in short breath holds. The right atrium was enlarged and divided by a perforated membrane between the crista terminalis and the inferior tricuspid annulus (Figure 1, A-C, arrowheads). A posterior apical atrial septal defect (Figure 1, B, arrow) with a diameter of $15 \mathrm{~mm}$ showed no cranial rim and right-to-left shunting. Phase-contrast MRI

From the Clinic for Congenital Heart Disease and the Institute for Magnetic Resonance Imaging a and the Clinic for Thoracic and Cardiovascular Surgery, ${ }^{\mathrm{b}}$ Heart and Diabetes Center Northrhine-Westfalia, Ruhr-University of Bochum, Bad Oeynhausen, Germany,

Received for publication March 4, 2006; accepted for publication March 15 , 2006.

Address for reprints: Samir Sarikouch, MD, Clinic for Congenital Heart Disease and Institute for Magnetic Resonance Imaging, Heart and Diabetes Center Northrhine-Westfalia, Ruhr-University of Bochum, Georgstra $\beta$ e 11, D-32545 Bad Oeynhausen, Germany (E-mail: ssarikouch@hdz-nrw.de).

J Thorac Cardiovasc Surg 2006;132:164-5

$0022-5223 / \$ 32.00$

Copyright @ 2006 by The American Association for Thoracic Surgery doi:10.1016/j.jtcvs.2006.03.031 stroke volume measurements in the ascending aorta $(73 \mathrm{~mL})$ and the main pulmonary artery $(59 \mathrm{~mL})$ quantified the right-to-

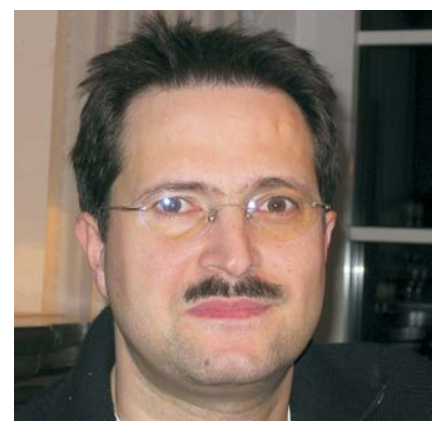

Dr Sarikouch left shunt as $18 \%$ of Qs, even at rest.

Cerebral MRI disclosed small postembolic defects in the left caudate nucleus and in both cerebellar hemispheres

Intraoperative situs (Figure 1,D) confirmed the diagnosis, revealing a sail-like membrane from the crista terminalis to the posterior-inferior tricuspid annulus dividing the right atrium into a posterior part with the apical atrial septal defect, receiving the venous return from the superior and inferior vena and the coronary sinus, and a ventral part of the right atrium with the normal tricuspid valve. The membrane showed 2 perforations with diameters of $1.5 \mathrm{~cm}$ at each vena caval orifice, which allowed venous blood to reach the right ventricle. The membrane was resected and the atrial septal defect was closed with an autologous pericardial patch. The patient had an uncomplicated postoperative course (with an oxygen saturation of $98 \%$ ) and was discharged 6 days later.

\section{Discussion}

Cor triatriatum dextrum is a rare congenital anomaly caused by remnants of the right sinus venosus valve. ${ }^{1}$ Failure in the regression process of the cranial part of this sinus venosus valve leads to membranes attached to the crista terminalis, as present in our case. Malformations of the lower embryologic valve parts result in a Chiari network or prominent eustachian or thebesian valves, which might eventually become large enough that they can even cause right ventricular outflow obstruction by prolapsing through the tricuspid valve. ${ }^{2,3}$

Cor triatriatum dextrum can present as an isolated malformation or as a part of complex right-sided heart defects. ${ }^{4}$ Right ventricular inflow obstruction and, in case of an associated atrial septal defect, signs of a right-to-left shunt can be discrete so that the diagnosis might be difficult and even be missed at cardiac surgery for other procedures. ${ }^{5} \mathrm{MRI}$ is a sensitive and noninvasive tool in the diagnosis of atrial malformations.

\section{References}

1. Abdulla R, Blew GA, Holtermann MJ. Cardiovascular embryology. Pediatr Cardiol. 2004;25:191-200.

2. Thomka I, Bendig L, Szente A, Arvay A. Cor triatriatum dextrum simulating right ventricular myxoma and pulmonary stenosis. Thorac Cardiovasc Surg. 1983;31:114-6.

3. Mahy IR, Anderson RH. Division of the right atrium. Circulation. 1998;98:2352-3.

4. Trento A, Zuberbuhler JR, Anderson RH, Park SC, Siewers RD. Divided right atrium (prominence of the eustachian and the thebesian valves). J Thorac Cardiovasc Surg. 1988;96:457-63.

5. Wyss E, Amman P, Rickli H, Jenni R. Cor triatriatum bei einem erwachsenen Patienten. Z Kardiol. 1998;87:891-3. 

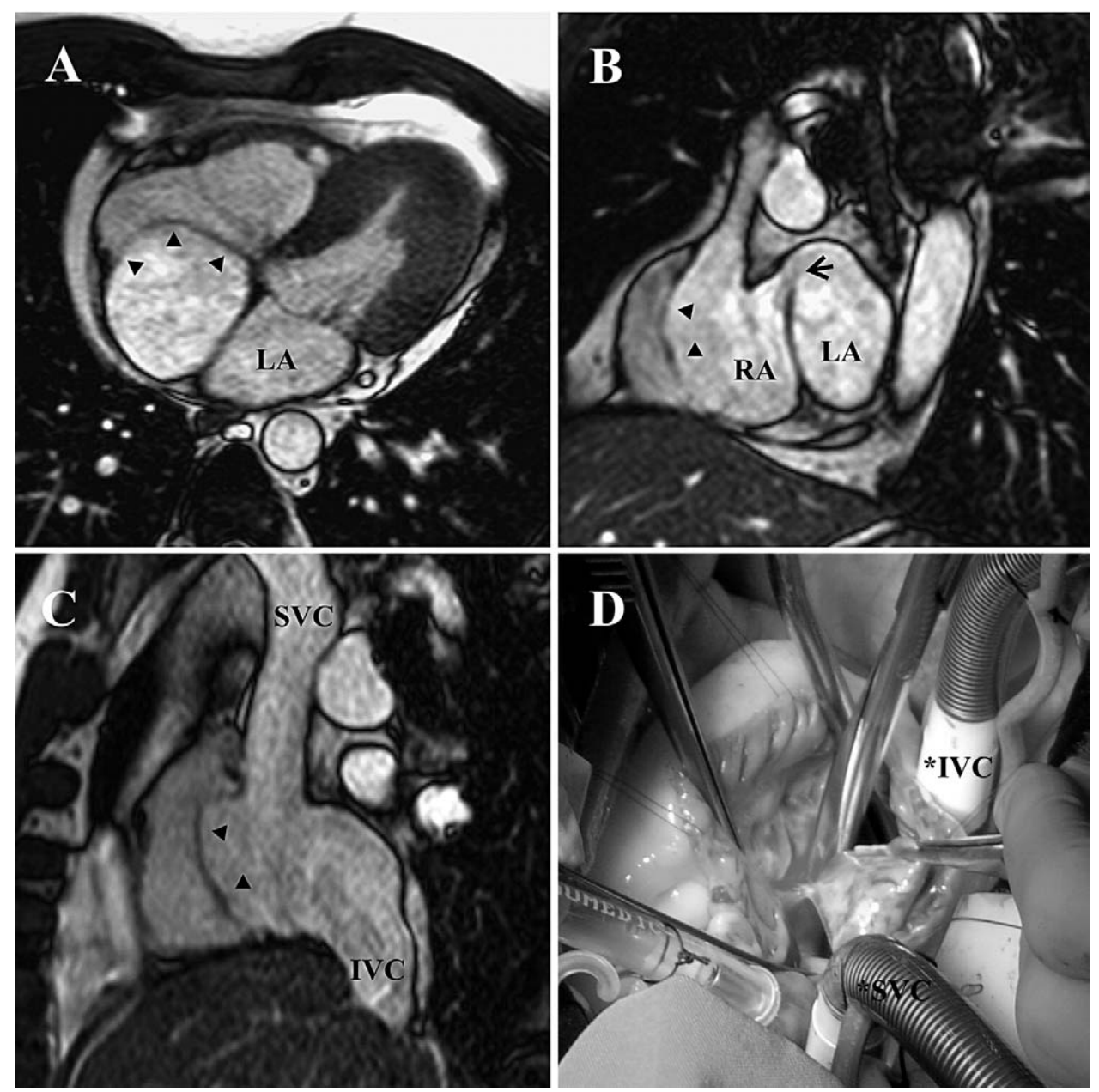

Figure 1. A, Balanced turbo field echo sequence (b-TFE) slice with a 4-chamber view showing the division of the right atrium by a membrane (arrowheads) close to the tricuspid valve. $L A$, Left atrium. $B$, Coronal b-TFE slice demonstrating the lateral aspect of the dividing membrane (arrowheads) at the crista terminalis and the posterior septal defect (arrow) between the left atrium (LA) and the right atrium (RA). C, Sagittal b-TFE slice shows the division of the right atrium (arrowheads) in a dorsal part, which receives the inferior vena cava (IVC) and the superior vena cava (SVC), and a smaller ventral part. D, Intraoperative situs demonstrating the membrane (scissor tip) dividing the right atrium and the 2 perforations with a diameter of $1.5 \mathrm{~cm}$, each representing the only inflow to the ventral part of the right atrium and the tricuspid valve. The posterior atrial septal defect is not visible. ${ }^{*}$ SVC and IVC mark the venous canulas in the superior and inferior vena cava. 